\title{
PONTEVEDRA (1901-1924)
}

\section{Paulino Aparicio Moreno}

IES Frei Martín Sarmiento (Pontevedra)

Desfilan por este artículo las diez compañías más prestigiosas que visitaron la ciudad de Pontevedra durante los años 1901-1924. Su presencia nos descubre una rica liturgia social desarrollada alrededor del altar teatral.

La principal diversión del público pontevedrés, como el de otros lugares, seguía siendo el teatro, a pesar del auge del cinematógrafo y de las variedades. Las clases más acomodadas acudían a él con la finalidad de ver $y$, especialmente, de ser vistas.

Esta vida teatral es una faceta importante del ambiente cultural de la vetusta Pontevedra de comienzos de siglo. Gracias al mejor conocimiento de dicho periodo, este panorama lejano hoy se nos antoja próximo y familiar.

Antes de centrarnos en el tema primordial de esta investigación, mostraremos algunos datos teatrales importantes que van empedrando el camino a las compañías. 
La primera noticia teatral que tenemos data de finales del siglo XVI, cuando la compañía de comedias de Juan Hurtado solicita permiso del Ayuntamiento para actuar en la ciudad.

La primera obra con título conocido es La muerte del Cid Ruy Díaz, escenificada, en 1595, por la compañía de Hernando de Navarrete.

Durante los siglos XVI, XVII y XVIII, las representaciones se hacían al aire libre, corrales o en inmuebles adaptados a tal fin. En 1753, el Padre Sarmiento afirma que Pontevedra no tenía «Casa de Comedias».

Tras varios intentos frustrados, la ciudad llegó a tener el primer teatro estable: el de la Plaza de Teucro. Su vida duró 30 años, de 1848 a 1878. Era un recinto humilde, incómodo y pequeño.

Pontevedra necesitaba un teatro digno y éste llegó con la construcción, no exenta de grandes contratiempos, del Teatro del Liceo. Un teatro de categoría, de piedra, hecho «a la italiana», para acoger sobre todo a la burguesía. Fue inaugurado, el día 2 de agosto de 1878, por la compañía de Miguel Cepillo, que subió a escena $E l$ esclavo de su culpa, de Juan Antonio Cavestany. La entrada de paraíso costó 1 peseta. En 1906, cambia de nombre y pasa a llamarse Teatro Principal, como en la actualidad. Hoy en día es un teatro nuevo, pues un incendio, ocurrido en la madrugada del 15 de abril de 1980, lo destruyó, dejando en cenizas 102 años de recuerdos y pasiones.

El miércoles, 25 de julio de 1900, a las 21:00 horas, abre sus puertas el Circo-Teatro, trabajando la compañía ecuestre de Luigi Cardinale.

Como teatro se inauguró el domingo 2 de septiembre de ese mismo año, a las 21:00 horas, por la compañía cómica de Juan Espantaleón, que estrenó La ducha, de Mariano Pina Domínguez, y La reja, de los hermanos Quintero. La entrada general se pagó a 50 céntimos.

Las diferencias con el Teatro Liceo son abismales. El Circo-Teatro viene a ser un barracón de madera, destinado especialmente a las clases populares, lo que conlleva precios más baratos. Aunque su creación estuvo acompañada de grandes polémicas, se mantuvo en pie durante 24 años. El sábado 26 de abril de 1924 fue completamente derruido, desapareciendo con él una importante parcela del ocio pontevedrés.

Otros lugares teatrales de menor importancia fueron:

El Teatro «Guignol»: barracón de madera, construido en la Alameda, en el verano de 1903. 
El Petit Palais: inaugurado el jueves 16 de febrero de 1911, por la noche. En 1913 fue adquirido por la Juventud Antoniana.

El nuevo teatrito de la Sociedad Recreo de Artesanos: abrió sus puertas el 26 de junio de 1912, a las 21:30 horas.

De los teatros citados, el Teatro Principal y el Circo-Teatro son los dos más importantes durante la época 1901-1924. En ellos representaron las compañías de más renombre de la época y los mejores actores.

Las compañías más numerosas fueron las Cómico-Dramáticas y las de menor presencia las Lírico-Dramáticas. No era frecuente ver a compañías especializadas en obras dramáticas y líricas a la vez. Las de ópera tampoco son muy abundantes, debido a los precios elevados y al poco fervor popular hacia dicho género.

Las compañías profesionales prestigiosas se caracterizan por tener una gran estabilidad, numeroso personal, rico vestuario y variado decorado.

La que más hizo para dignificar la escena fue la de María Guerrero y Fernando Díaz de Mendoza, que procuró dar mayor propiedad y realismo a la representación, acompañándola de un alto y exquisito lujo. Fueron los primeros que compraron muebles propios para la escenificación e incorporaron el cargo de mueblista para tal fin. Posteriormente, las compañías principales seguirían este ejemplo.

Frente a éstas, encontramos otras más humildes, algunas de ellas con multitud de problemas internos como la de Gustavo Belza y Eugenio Casals, la de Fernando Viñas y Lorenzo Sola o la de Miguel Lía.

La gran mayoría de las compañías venían de Madrid y generalmente traían los estrenos que habían subido a las tablas en dicha capital. El primer actor o la primera actriz eran el centro sobre el que seguían formándose las compañías, a las que solían dar nombre, desempeñando generalmente el papel de director de las mismas. Esto motiva que haya un liderazgo exclusivista, teniéndose que acomodar el resto de los actores a un papel secundario, para no hacerles sombra. El lucimiento personal guiaba el afán de estos divos, pudiéndose hablar de un estilo personalista.

Este exclusivismo llega incluso hasta el punto de seleccionar los papeles a su medida. Algunos autores escribían pensando en un actor o actriz determinados. Es el caso, por ejemplo, del dramaturgo José Echegaray, respecto a María Guerrero. 
En líneas generales, podemos decir que el panorama de las compañías da muestras de una sensible mejoría, aunque de manera lenta y tímida.

En cuanto a las compañías de aficionados, diremos que destacan las de Pontevedra capital, especialmente la Juventud Antoniana y la Sociedad Artística. Un primero de mayo, unos Carnavales, una acción con fines benéficos... servían a estos aficionados para llevar al escenario obras generalmente breves, demostrando sus aceptables dotes para la representación. El público acudía masivamente a estos actos, que recibían por parte de la crítica elogios muy meritorios. Como puede deducirse, la afición al teatro en la ciudad era muy grande.

Entre las compañías profesionales, las diez más importantes fueron:

1) Compañía Dramática de Enrique Borrás.

2) Compañía Dramática de Carmen Cobeña.

3) Compañía Dramática de María Guerrero y Fernando Díaz de Mendoza.

4) Compañía Cómico-Dramática de María Fernanda Ladrón de Guevara y Rafael Rivelles.

5) Compañía Dramática de Francisco Morano.

6) Compañía Dramática de Rosario Pino.

7) Compañía de Zarzuela y Verso de Loreto Prado y Enrique Chicote.

8) Compañía Cómico-Dramática del Teatro de la Comedia de Madrid.

9) Compañía Cómico-Dramática del Teatro Español de Madrid.

10) Compañía Dramática de Margarita Xirgu.

El orden seguido para la clasificación ha sido el alfabético. Veamos a continuación los componentes y el repertorio de las mencionadas compañías, el número de representaciones de cada obra y si fueron estreno. Las letras $\mathrm{r}$ y $\mathrm{E}$, que van entre paréntesis, así lo indican. También aclaramos las siguientes abreviaturas utilizadas: 
CJC: Colección José Casal.

DP: El Diario de Pontevedra.

MP: Museo de Pontevedra.

P: Progreso.

\section{1) Compañía Dramática de Enrique Borrás}

Trabajó entre el 2 y el 10 de julio de 1916 en el Teatro Principal.

\section{a) Componentes:}

\section{Actrices:}

Anaya, María

Camarero, Asunción.

Castejón, Micaela

Galiana, Matilde

Gómez, María: primera actriz

Garcés, Mercedes

Pacheco, Elvira

Pacheco, María

Vivero, Asunción

Zamora, Josefina

\section{Actores:}

Blanca, Eduardo

Borrás, Enrique: primer actor

Brasal López, Luis

Cantalapiedra, Enrique

Carmona, Menandro

Catalá, Juan

Domínguez, Luis

Fernández, Juan

Martín, Manuel

Nicolau, Carlos

Ozores, Mariano

Requena, Rafael

Vico, José

Zarco, Francisco

\section{Otros:}

Apuntadores: José Hidalgo y Ricardo Vico

Maquinista: José Rodríguez y Arturo Alonso

Electricista: Miguel González» (DP, 15-VI-1916: 2).

\section{b) Repertorio de obras interpretadas:}

Alcalde de Zalamea, El (1r)

Cena de burlas, La (1r, E)

Gran galeoto, El (1r)

Místico, El (1r) 
Muerte civil, La (1r, E)

Tierra baja (1r)

Vida es sueño, La (1r).

2) Compañía Dramática de Carmen Cobeña

a) Componentes:

- Primera estancia (26 y 30 de octubre de 1908: Circo-Teatro):

Actrices:

Adsuar, Concepción

Ahijón, María Luisa

Álvarez, Josefina

Anaya, María

Cobeña, Carmen

Iraguirre, Consuelo

Luna, Isabel

Llorente, Matilde

Valdemoro, Carmen

Villabona, Mercedes
Actores:

Almansa, José

Cobeña, Benito

Cobeña, Rafael

Comes, Francisco

Llorens, Federico

Manso, Ricardo

Perrín, Manuel

Requena, Rafael

Ruiz Tatay, Leovigildo

Soto, Carlos

\section{Otros:}

Apuntadores: José Charcán y Alfredo Ruiz

Representante de la compañía: Manuel Domínguez (CJC, MP).

- Segunda estancia (del 1 al 4 de marzo de 1919: Teatro Principal):

Actrices:

Cobeña, Carmen

Cuevas, María

De la Fuente, Orencia

Eladio, Teresa

Fernández de Córdoba, Francisca

G. Moya, Luz

Gil Andrés, Juana

Hurtado, Manuela

Manuel, Rosario

Zaldívar, María

\section{Actores:}

Babe Botana, Andrés

Calavera, Francisco

Cobeña, Benito

Cobeña, Rafael

Gómez, Andrés

Gómez de la Vega, Alfredo

López Brasal, Luis

Muñoz, Miguel

Nogales, Manuel

Ortiz, Antonio 
Zeda, Emilia

$$
\begin{aligned}
& \text { Ventura, José } \\
& \text { Viñas, Constante } \\
& \text { Zori, Antonio. }
\end{aligned}
$$

\section{Otros:}

Apuntadores: Vicente Serrano, Mariano Artiaga y Manuel Perrín Maquinista: Santiago Mascaraque

Atrecista: Antonio Ruiz

Representante: Ricardo Miranda (DP, 27-II-1919: 2).

b) Repertorio de obras interpretadas:

- Primera estancia ( 26 y 30 de octubre de 1908: Circo-Teatro):

Las de Caín (1r, E)

Madre, La (1r, E).

- Segunda estancia (del 1 al 4 de marzo de 1919: Teatro Principal):

Cobardias (1r, E)

Esclavitud (2r, E)

Locura de amor, La (1r, E)

Milagro, $E l(1 \mathrm{r}, \mathrm{E})$

Pueblo dormido, El (1r, E).

3) Compañía Dramática de María Guerrero y Fernando Díaz de Mendoza

\section{a) Componentes:}

- Primera estancia (del 18 de julio al 2 de agosto de 1902: CircoTeatro):

\section{Actrices:}

Bofill, Encarnación

Bueno, Matilde

Cancio, María

Coy, Cecilia

Guerrero, María

Martínez, Julia
Actores:

Allen Perkins, Carlos

Barragán, Enrique

Buil, Vicente

Calle, José

Carsí, Felipe

Cirera, Alfredo 
Pérez, Teresa

Ruiz, Concepción

Segarra, Carmen

Socias, Laura

Valdivia, Ramona

Zaragoza, Jacinta
Díaz de Mendoza, Fernando

Díaz de Mendoza, Mariano

Díaz, Manuel

Guerrero, Ramón

Juste, Ricardo

Manchón, Antonio

Medrano, Luis

Miguel, Alberto

Robles, Juan

Ruiz Tatay, Hermenegildo

Soriano, José

Urquijo, Francisco

Villalonga, Fernando (CJC, $M P)$.

- Segunda estancia (del 1 al 3 de octubre de 1907: Teatro Principal):

Actrices:

Bárcenas, Julia

Bofill, Encarnación

Bremón, Dolores

Bueno, Matilde

Cancio, María

Cotera, Luisa

García, Luisa

Guerrero, María

Martínez, Francisca

Riquelme, Elena

Roca, Josefina

Salvador, Elena
Actores:

Carsí, Felipe

Cayuela, Francisco

Cirera, Alfredo

Codina, Pedro

Díaz, Manuel

Díaz de Mendoza, F.

Díaz de Mendoza, $M$.

Gil, Manrique

Guerrero, Ramón

Juste, Ricardo

Medrano, Luis

Santiago, José

Soriano Viosca, José

Urquijo, Francisco

Vargas, Ricardo» (CJC, $M P$ ).

— Tercera estancia (7 y 8 de junio de 1917: Teatro Principal):

Actrices:

Adriani, María

Bitano, María

Bofill, Encarnación

Bueno, Matilde

Cancio, María
Actores:

Capilla, José

Carsi, Felipe

Cirera, Alfredo

Cirera, Felipe

Corona, Fernando 
Carbonell, Carmen

Carbonell, María Teresa

Guerrero, María

Hermosa, María

Ladrón de Guevara, M. ${ }^{a}$ Fernanda

Ruiz Moragas, Carmen

Salvador, Elena

Torres, Avelina
Defauce, Félix

Díaz de Mendoza, Fernando

Díaz de Mendoza, Mariano

Díaz de Mendoza y Guerrero, C.

Díaz de Mendoza y Guerrero, F.

Guerrero, Ramón

Juste, Ricardo

Labra, R. M. ${ }^{\mathbf{a}}$

Medrano, Luis

Palanca, Francisco

Santiago, José

Urquijo, Francisco

Valenti, Emilio

Vargas, Ricardo (DP, 14-V-1917: 2).

b) Repertorio de obras interpretadas:

- Primera estancia (del 18 de julio al 2 de agosto de 1902: CircoTeatro):

A cadena perpetua (1r, E)

Canto de la sirena, El (1r, E)

Estigma, El (1r, E)

Loco Dios, El (1r)

Malas herencias (1r, E)

Mi misma cara (1r, E)

Positivo, Lo (1r, E)

Sota de bastos, La (1r)

Vergonzoso en palacio, El (1r, E).

- Segunda estancia (del 1 al 3 de octubre de 1907: Teatro Principal):

Genio alegre, $E l(1 \mathrm{r})$

Ladrón, El (1r, E)

Mancha que limpia (1r).

- Tercera estancia (7 y 8 de junio de 1917: Teatro Principal):

Enemiga, La (1r, E)

Leona de Castilla, La (1r, E). 
4) Compañía Cómico-Dramática de María Fernanda Ladrón de Guevara y Rafael Rivelles

a) Componentes:

\section{Actrices:}

Ayala, Almudena

Cáceres, Juana

Cámara, Laura María

Collado, Carmen

Ladrón de Guevara, María Fernanda

Llano, Elvira

Llano, Soledad

Sánchez Ariño, Amalia

Satorres, Josefina
Actores:

Gentil, Antonio

Orduña, Juan de

Pizá, Francisco

Porredón, Fernando

Ramírez, Luis

Rivelles, Rafael

Rodrigo, Emilio

Rodríguez, Manuel

Vilches, Enrique

Zapico, María Ofelia.

\section{Otros:}

Apuntadores: Rufino López y Jesús Pastrana

Jefe maquinista: José Ruano

Gerente de la Compañía: Vicente Castilla (P, 27-II-1924: 2).

b) Repertorio de obras interpretadas

- Primera estancia (del 1 al 13 de marzo de 1924: Teatro Principal):

Aire de fuera (2r)

Amores y amorios (2r)

Bandido de la sierra, El (3r, E)

Currito de la Cruz (4r, E)

Felipe Derblay (2r)

Inmaculada de los Dolores, La (2r, E)

Loca aventura, La (2r, E)

Madrigal de la cumbre, El (2r)

Mala ley, La (1r)

Nido ajeno, El (1r)

Señorita Ángeles, La (1r, E)

Único señor, $E l(2 \mathrm{r}, \mathrm{E})$

Vueltas que da el mundo, Las (2r, E). 
- Segunda estancia (8 y 9 de abril de 1924: Teatro Principal):

Jaula de la leona, La (2r, E)

Mi hermano y yo (2r, E).

\title{
5) Compañía Dramática de Francisco Morano
}

Representó del 19 al 21 de julio de 1920 en el Teatro Principal.

a) Componentes:

Primer actor y director: Francisco Morano

Primera actriz: Amparo Fernández Villegas

Director artístico: Antonio Navarro

Actrices:Actores:

Pura Fernández Villegas

Eugenia Fraile

Carmen L. Lagar

Raquel Martínez

Ángeles Morano

Sofía Morano

Julia Sala

María Santoncha

Josefina Satorres

\author{
Francisco Calvera \\ Antonio Gentil \\ Luis Herrero \\ Francisco López Silva \\ Gonzalo Llorens \\ Marcial Morano \\ Fernando Porredón (H.) \\ Fernando Sala \\ Vicente Soler \\ Ángeles Somavilla \\ María Zarco.
}

\section{Otros:}

Apuntadores: Carlos Ávalos, Manuel Perrín y Felipe Gracia Jefe maquinista: Matías Rubio

Representante administrativo: Manuel Domínguez (DP, 12-VII1920: 3).

b) Repertorio de obras interpretadas:

Cardenal, El (1r)

En la mitad del corazón (1r, E)

Papá Lebonnard (1r, E). 
6) Compañía Dramática de Rosario Pino

a) Componentes:

- Primera estancia (del 3 al 5 de mayo de 1913: Teatro Principal):

Actrices:

Rafaela García

Consuelo León

María Marín

Isabel Mas

Pascuala Mesa

Guadalupe Muñoz Sampedro

Carlota Pazo

María Pazo

Rosario Pino

Adriana Robles

Concepción Robles

Josefina Satones

Dolores Valero

Isabel Zaldívar
Actores:

José Cañizares

Sixto Coduras

Luis Echaide

José García de Leonardo

Antonio Gentil

Antonio Lagos

Manuel Menéndez

Ricardo Miranda

Enrique Moreno

Juan R. Ginestal

Nicolás Rodríguez

Alberto Serrano

Manuel Soto

Emilio Valenti.

Otros:

Apuntadores: Luis Azaña, Rafael García y Sixto Coduras

Secretario: Antonio Vico (DP, 21-IV-1913: 2).

- Tercera estancia (del 19 al 23 de junio de 1914: Teatro Principal):

Actrices:

Rosario Pino

Conchita Robles

Eloísa Parejo

Adela Garzón

Carlota Pazo

Isabel Mas

Florentina Montosa

Robles Pérez

Pazos Hernández

Joaquina Almarche

\section{Actores:}

Emilio Valenti

Fausto Otero

Fernández de la Somera

Horacio Socias

Manuel Vigo

Antonio Lagos

José Cañizares

Nicolás González

Enrique Pérez

Juan García (DP, 15-VI-1914: 2). 
b) Repertorio de obras interpretadas:

- Primera estancia (del 3 al 5 de mayo de 1913: Teatro Principal):

Comida de las fieras, $L a(1 \mathrm{r}, \mathrm{E})$

Nena Teruel (1r, E)

Noche del sábado, La (1r, E).

- Segunda estancia (12 y 13 de mayo de 1913: Teatro Principal):

Divorciémonos (1r)

Genio alegre, $E l(1 \mathbf{r})$

Sacrificios (1r, E).

- Tercera estancia (del 19 al 24 de junio de 1914: Teatro Principal):

Alma triunfante (1r, E)

Amores y amoríos (1r)

Malquerida, La (1r)

Malvaloca (1r)

Orgullo de Albacete, El (Loute) (1r, E)

Primavera en otoño (1r)

Rosas de todo el año (1r, E)

Sangre gorda $(1 \mathrm{r}, \mathrm{E})$

Sorpresas (1r, E).

- Cuarta estancia (30 de junio de 1920: Teatro Principal):

Corazón en la mano, El (1r, E)

Flor de la vida, La (1r, E).

- Quinta estancia (19 y 20 de julio de 1924: Teatro Principal):

Concha, la limpia $(3 \mathrm{r}, \mathrm{E})$.

7) Compañía de Zarzuela y Verso de Loreto Prado y Enrique Chicote

Representó del 27 de abril al 3 de mayo de 1921 en el Teatro Principal.

\section{a) Componentes:}

\section{Actrices:}

Amalia Anchorena

María Luisa Arias

\section{Actores:}

Julio Castro

Enrique Chicote 


$\begin{array}{ll}\text { Ángeles Campos } & \text { José Delgado } \\ \text { María Clemente } & \text { Eduardo Díaz de la Vega } \\ \text { Matilde Franco } & \text { Ramón Gotós } \\ \text { Pilar Gandía } & \text { Carlos Henche } \\ \text { Mercedes Garcelán } & \text { José M. Rey } \\ \text { Paula Martín } & \text { Ricardo Manso } \\ \text { Rosario Martínez } & \text { José Ortiz } \\ \text { Luisa Melchor } & \text { José Ponzano } \\ \text { Julia Melero } & \text { Rodolfo Recober } \\ \text { Goya, Mir } & \text { Augusto Rodríguez Aria } \\ & \text { María Montenegro } \\ & \text { Loreto Prado } \\ & \text { Elisa Román. }\end{array}$

\section{Otros:}

Maestros directores y concertadores: Pedro Badía y Roque S. Redondo

Apuntadores: Antonio Povenado y Francisco Giménez

Maquinistas: José y Manuel Martínez

Representante de la compañía: Julio Poveda (DP, 21-IV-1921: 2).

\section{b) Repertorio de obras interpretadas:}

Aventura de Colón, La (2r, E)

Chicos de la escuela, Los (2r)

Colilla IV (2r, E)

Ellas (2r, E)

Gente menuda (2r, E)

Granujas, Los (2r)

Liga de las naciones, $L a(2 \mathrm{r}, \mathrm{E})$

Machacante, El (2r, E)

Mi sobrino Fernando (2r, E)

Perros de presa, Los (2r).

8) Compañía Cómico-Dramática del Teatro de la Comedia de Madrid

a) Componentes:

- Primera estancia (del 30 de junio al 17 de julio de 1905: CircoTeatro): 


\section{Actrices:}

Caro, Alejandrina

Catalá, Concepción

Colorado, Margarita

Luna, Isabel

Pérez Vargas, Mercedes

Pino, Rosario: primera actriz

Roca, Josefina

Torres, Evelina

Toscano, Rosario

Vigil, Ernesta

Zamora, Victoria

\section{Actores:}

Balaguer, Juan: primer actor cómico Baylés, Mariano

Borrás, Enrique: primer actor

González, Manuel

Gonzálvez, Federico

Lliri, Juan

Manrique, Luis

Mora, Salvador

Ruiz-Tatay, Leovigildo

Sala, Ángel

Vico, José.

\section{Otros:}

Apuntadores: Pérez Liquiñano, Ambrosio y Ruiz, Antonio

Maquinistas: Francisco López y José Ramos

Electricista: Juan Morcate (CJC, $M P$ ).

- Segunda estancia (del 25 al 27 de agosto de 1919: Teatro Principal):

\section{Actrices:}

Alba, Irene

Andrés, Carmen

Caba, Irene

Carbone, Adela

Jiménez, Josefa

León, Carmen

Lobo, Pilar

Mesa, Pascuala

Redondo, Aurora

Rey, María

Villa, Carmen

\section{Actores:}

Asquerino, Mariano

Bonafé, Juan

Caba, Manuel

Espantaleón, Juan

García, Fortunato

Insúa, Manuel

Moreno, Enrique

Pereda, Francisco

Riquelme, Antonio

Roa, Joaquín

S. del Valle, Vicente

Zorrilla, Pedro.

\section{Otros:}

Apuntadores: Francisco Méndez, Manuel Agusif y Rafael Sala

Maquinistas: Valentín González y Antonio Ramos

Guardarropa: Gregorio Hermana

Electricista: José Pérez (DP, 16-VIII-1919: 2). 
b) Repertorio de obras interpretadas:

- Primera estancia (del 30 de junio al 17 de julio de 1905: CircoTeatro):

Alcalde de Zalamea, El (1r, E)

Asistentes, Los (1r)

Loca de la casa, La (1r, E)

Mar y cielo (1r, E)

Marido pintado, El (1r, E)

Místico, El (1r)

Parada y fonda (1r, E)

Primo Prieto (1r, E)

Sin querer (1r, E)

Tierra baja $(1 \mathrm{r}, \mathrm{E})$

Viejos, Los (1r, E).

- Segunda estancia (del 25 al 27 de agosto de 1919: Teatro Principal):

Casa de la Troya, La (2r, E)

Venganza de Don Mendo, La (1r, E).

9) Compañía Cómico-Dramática del Teatro Español de Madrid

Trabajó entre el 6 y el 11 de agosto de 1912 en el Teatro Principal.

a) Componentes:

Primera actriz: Matilde Moreno

Primer actor: Ricardo Puga

Actrices:

María Barral

Concepción Bermejo

Luisa Calderón

Micaela Castejón

Concepción Estrella

Nieves Fernández

Clotilde Guerra

Elisa Méndez

Celia Ortiz
Actores:

Pedro Cabré

Rafael Calvo

Rafael Cobeña

Fernando Estrella

Delfín Jerez

Alejandro Maximino

Germán de Molina Sylas

José Mora

Agustín Povedano 
Julia Pérez

Pastor Roncallo Ángel Sepúlveda (DP, 5-VIII-1912: 2).

b) Repertorio de obras interpretadas:

Escuela de las princesas, La (1r)

Jimmy Samson (1r, E)

Malvaloca (1r)

Matrimonio interino, El (1r)

Rosas de otoño (1r).

10) Compañía Dramática de Margarita Xirgu

Actuó del 3 al 7 de agosto de 1921 en el Teatro Principal.

a) Componentes:

Actrices:

Álvarez-Segura, Amparo

Arrate, Carmen

Bru, María

Cáceres, Juana

Carbonell, Carmen

Cebrián, Pilar

Clavijo, Ángela R. de

Rivas, María de las

Santaularia, Adela

Xirgu, Margarita
Actores:

Agudín, Luis P.

Álvarez, Carlos

Codina, Víctor

González, Pedro

Lecio, José

Muñoz, Alfonso

Ortín, Miguel

Perchicot, Nicolás D.

Ribas, Modesto

Rivero, José

Samsó, Roberto.

\section{Otros:}

Apuntadores: José Bofill, Joaquín Llacer y Roberto Zappino

Maquinista: Mariano Jaime

Mueblista: Rafael Arroyo (DP, 1-VIII-1921: 3).

b) Repertorio de obras interpretadas:

Carmen (1r, E)

Extraña, La (1r, E)

Fedora (1r) 
Mal que nos hacen, El (1r)

Ramo de locura (1r, E).

Como hemos podido comprobar, todas las compañías, excepto la de Loreto Prado y Enrique Chicote que se anuncia de Zarzuela y Verso, representan obras del teatro declamado. Si observamos detenidamente en sus repertorios, vemos que en los escenarios pontevedreses prevalecen obras contemporáneas a su puesta en escena. La cercanía entre teatro escrito y representado resulta evidente, aunque aparezca alguna producción de nuestro teatro clásico. Este tipo de piezas refleja fielmente el teatro de éxito de la época estudiada. Es decir, el comercial. No encontramos creaciones de los autores denominados renovadores como Unamuno, Valle-Inclán, Lorca...

Entre los títulos más celebrados, encontramos Currito de la Cruz, comedia en tres actos y un epílogo, de Manuel Linares Rivas, adaptación de la novela de Pérez Lugín, con cuatro representaciones; El bandido de la sierra, drama en tres actos y un epílogo, en verso, de Luis Fernández Ardavín, Amores y amoríos, comedia en cuatro actos, de los hermanos Quintero y Concha, la limpia, comedia en tres actos, también de los Quintero, con tres. Estos dos últimos autores fueron los más representados en Pontevedra.

Si tenemos curiosidad por saber las compañías con más obras escenificadas, la siguiente relación así nos lo aclara:

Cía. de Rosario Pino............................................. 18 obras

Cía. de Ladrón de Guevara..................................... 15 "

Cía. de M. a Guerrero.............................................. 14 "

Cía. del Teatro de la Comedia de Madrid............... 13

Entre las compañías que más veces visitaron la ciudad se encuentran la de Rosario Pino, que lo hizo en cinco ocasiones, y la de M. $^{\mathbf{a}}$ Guerrero, en tres.

En cuanto a la crítica recibida por parte de la prensa pontevedresa, diremos que predomina una línea encomiástica en sus reseñas. Los grandes actores y actrices acaparaban la atención de los críticos, que les dedicaban los artículos más extensos y elogiosos.

Las compañías ya han terminado sus repertorios. Bajemos el telón y soñemos de nuevo. 